$\xi=2$ 잘

\title{
Reliability of digital radiographs in measuring the vertical and horizontal dental implants' dimensions a retrospective study
}

\author{
Dr. Rakan Shaheen ${ }^{1}$ *, Dr. Muna Binladin ${ }^{2}$, Dr. Alanoud Bin Muammar ${ }^{2}$, Dr. Shuaa Alonazi ${ }^{2}$, \\ Dr. Amjad Alharbi ${ }^{2}$, Dr. Bader Bamousa ${ }^{1}$, Dr. Ahmed Alahmari ${ }^{3}$ \\ ${ }^{I}$ MSc in Periodontics, Preventive Department, College of Dentistry, Riyadh Elm University, Saudi Arabia \\ ${ }^{2}$ BDS, Dental Intern, College of Dentistry, Riyadh Elm University, Saudi Arabia \\ ${ }^{3}$ MSc in Periodontics, Department of Periodontics, Director of Khamis Mushait Specialty Dental Center, Saudi Arabia \\ *Corresponding author E-mail: rakan.s.shaheen@gmail.com
}

\begin{abstract}
Introduction: Digital radiography can take accurate measurements. In implant dentistry, intraoral periapical (PA), bite-wing (BW) and panoramic (OPG) radiographs are being used, although it had limitations of distortion. This research aims to assess the accuracy of digital radiography in measuring dental implants and to evaluate the SIDEXIS software's reliability in measuring their dimensions. Materials and Methods: Over 192 implants from 316 radiographs were selected. All radiographs were analyzed using SIDEXIS software. Statistical analysis was performed using SPSS.

Results: Statistically significant differences $(\mathrm{P}<0.5)$ between the actual heights and widths compared to the measured radiographic dimensions. Over all the distortion was greatest in OPGs. The magnifications were $2.48 \mathrm{~mm}$ in height and $0.82 \mathrm{~mm}$ in width for OPGS, $0.17 \mathrm{~mm}$ in width for BWs while in PAs it was $1.37 \mathrm{~mm}$ in height and $0.156 \mathrm{~mm}$ in width. The magnification was more in maxilla for PAs and OPGs. Anteriorly the PAs had the greatest magnification $(2.16 \mathrm{~mm})$, and OPGs had the greatest at $(3.03 \mathrm{~mm})$ in height posteriorly. Conclusion: Digital OPG, PA and Bitewing radiographs are reliable for performing dimensions linear measurements for implants, and PAs have the highest precision. Additionally, SIDEXIS software provided accurate results and can be considered a reliable method for implants' assessment.
\end{abstract}

Keywords: Digital; Sidexis; Implant; Measurement; Accuracy.

\section{Introduction}

Digital radiography has a variety of benefits; one of which is being able to take accurate measurements. In implant dentistry, Intraoral radiographs offer the best resolution among all the imaging modalities. Several factors might affect the image quality, including objectfilm distance, controlled alignment of the film, and the object and imaging source (Sahiwal, Woody et al. 2002).

Due to the availability and accessibility of panoramic (OPG) radiographs it have been used widely in implant treatment planning (McDavid, Dove et al. 1993), Although it had limitations of distortion, superimposition with variability in inter-examiner agreement (Carmichael, Hirschmann et al. 2000). Even when properly taken; the actual object size gets enlarged by about 15 - 25\% (Sanderink, Visser et al. 1991). (Samawi and Burke 1984) conclude that canine and premolar regions are the greatest in magnification and the third molar region is the least.

(Machtei, Zigdon et al. 2010) measured the distance from the apex of a pilot drill to the roof of the inferior alveolar canal on a digital OPG and compared it with the readings of an ultrasonic device used to measure the distance from the bottom of the osteotome preparation to the canal. They found a strong and highly significant correlation between the two measurement methods. By using a digital OPG, (Kim, Park et al. 2011) evaluated the rate of magnification in dental implants, they found that the mean radiological magnification in the mandibular premolar and mandibular molar region was 1.26 and 1.25 respectively.

(Park 2010) reported that the mean magnification of mandibular implants using digital OPGs in the premolar region and the molar region was 1.31 and 1.27 respectively and concluded that to evaluate the available bone height for posterior mandibular implants, digital OPGs were sufficiently accurate.

Full information of the trabecular pattern and the relationship of anatomic structures in the implant site area was provided by Intraoral radiographs. Intraoral radiography has several advantages; being low-cost, easily available, in most cases well tolerated by patients, and provide high-resolution images of the implant site. Meanwhile, there are some disadvantages which include inconstant imaging geometry, distortions that are inherent in intraoral radiography, and lack of cross-sectional information, which is important in implant site evaluation (Tyndall and Brooks 2000). In many cases, the radiographs along with proper clinical examination can be sufficient to determine the size and position of implants if distortion is accounted for (Kayal 2016). 
This study aimed to assess the accuracy of digital radiography in measuring dental implants and to evaluate the SIDEXIS software's reliability in measuring their vertical and horizontal dimensions.

\section{Materials and methods}

OPGs, periapical (PA) radiographs and bite-wing (BW) radiographs were selected from patients' records database at the Faculty of Dentistry, Riyadh Elm University after obtaining approval from the Research Ethics Committee. Over 190 implants placed anteriorly and posteriorly were included. The Nobel implants system (Nobel Biocare, Kloten, Switzerland) was the system of choice for this study. Radiographs with any artifact that affects the implant measurement were excluded. All radiographs were analyzed by using the SIDEXIS XG Software (Sirona Dental System, Bensheim, Germany) and the examiners were blinded to the actual size of the dental implants. Inter-examiner and intra-examiner reliability were assessed and finalized prior to the data collection.

The selected PA and BW radiographs were obtained with the Heliodent Plus X-ray unit (Dentsply, Sirona, Bensheim, Germany) operating at $60 \mathrm{kVp}$ with $0.08 \mathrm{~s}$ exposure time. With the sensor used being the film XIOS XG Supreme Sensor D3495 (Sirona Dental System, Bensheim, Germany). And the selected OPGs were obtained using the Orthophos XG 5DS x-ray unit (Sirona Dental System, Bensheim, Germany), and the equipment were adjusted to $64 \mathrm{kVp}$ and $8 \mathrm{~mA}$ with $14.1 \mathrm{~s}$ exposure time. Two examiners were calibrated to the measuring technique on the SIDEXIS XG Software by measuring with a mouse driven caliper, on MSI AIO PC (16:9 aspect ratio, 1920x1080 resolution) display screen. The vertical dimension was measured from the upper limit (coronal collar) of the dental implant to the most apical point of the implant, and the horizontal dimension was measured on the most coronal part of the dental implant from the most distal point to the most mesial point. Statistical analysis was performed using Statistical Package for the Social Sciences SPSS $\odot$.

\section{Results}

Over 19 2implants from 316 radiographs distributed between PAs, BWs and OPGs were analyzed. of these, 9 6were placed in the maxilla and 96 implants were placed in the mandible. The number of implants placed in the anterior and posterior regions were $72,120 \mathrm{im}-$ plants respectively. Implants' diameters were $3.5 \mathrm{~mm}$ (800 implants), $4.3 \mathrm{~mm}$ (780 implants), and $5 \mathrm{~mm}$ ( 250 implants). Their lengths were $8 \mathrm{~mm}$ (27 implants), $10 \mathrm{~mm}$ (106 implants), $11.5 \mathrm{~mm}$ (28 implants), and $13 \mathrm{~mm}$ (31 implant). The intra-observer and inter-observer agreement for the measurement of radiographic dimensions was 0.81 using Cronbach's alpha which shows a good reliability of the measured dimensions.

The width and length of the dental implants were measured on digital PA, BW, and OPG radiographs using the SIDEXIS XG Software and were compared to the actual sizes of these implants. Examining the implants placed in all regions collectively revealed statistically significant differences $(\mathrm{P}<0.5)$ between the actual heights and widths compared to the measured radiographic dimensions. [Tables 1,2 , $3,4]$

Taking a deeper look at the data by examining each region separately revealed that for the anterior and posterior regions; the distortion was greatest in OPGs, then BWs and finally PAs. The mean magnifications for these three types of radiograph were $2.48 \mathrm{~mm}$ in height and $0.82 \mathrm{~mm}$ in width for OPGS, $0.17 \mathrm{~mm}$ in width for BWs while in PAs it was $1.37 \mathrm{~mm}$ in height and $0.156 \mathrm{~mm}$ in width.

In OPGs the mean magnification of the posterior region was greater than the anterior, with being $2.74 \mathrm{~mm}$ in height, and $0.97 \mathrm{~mm}$ in width for implants placed in the posterior region. While comparing anterior and posterior regions in PAs; the anterior implants had greater mean magnification in height $(1.93 \mathrm{~mm})$, but as for the mean magnification in width, the posterior region was greater than the anterior (0.188mm). [Table 1]

Table 1: Dimensional Magnifications between Anterior and Posterior Implants

\begin{tabular}{|c|c|c|c|c|c|}
\hline Height Magnification on Anterior PAs & $\begin{array}{l}\text { Mean } \\
-1.92852\end{array}$ & $\begin{array}{l}\text { Std. Deviation } \\
1.40451\end{array}$ & $\begin{array}{l}\mathrm{t} \\
-10.090\end{array}$ & $\begin{array}{l}\mathrm{df} \\
53\end{array}$ & $\begin{array}{l}\text { Sig. } \\
.000\end{array}$ \\
\hline Width Magnification on Posterior PAs & -.18760 & .27106 & -4.894 & 49 & .000 \\
\hline Width Magnification on Posterior BWs & -.17146 & .24606 & -7.072 & 102 & .000 \\
\hline Height Magnification on Anterior OPGs & -2.26814 & .95242 & -18.292 & 58 & .000 \\
\hline Height Magnification on posterior OPGs & -2.73500 & .81239 & -23.805 & 49 & .000 \\
\hline Width Magnification on Posterior OPGs & -.96540 & .46869 & -14.565 & 49 & .000 \\
\hline
\end{tabular}

Different sizes of implants were examined, in PAs the implants with $13 \mathrm{~mm}$ lengths recorded the maximum mean height magnification $(1.65 \mathrm{~mm})$, and the implants with $3.5 \mathrm{~mm}$ widths had the maximum mean width magnification $(0.167 \mathrm{~mm})$. Regarding OPGs, the greatest mean height magnification was seen in implants with the length $11.5 \mathrm{~mm}(3.08 \mathrm{~mm})$ and implants with the width $5 \mathrm{~mm}$ showed the greatest mean width magnification $(0.96 \mathrm{~mm})$. whereas in BWs the greatest mean width magnification was recorded in implants with the width $3.5 \mathrm{~mm}(0.31 \mathrm{~mm})$. [Tables 2,3$]$

Table 2: Dimensional Magnifications between Various Implant Heights

\begin{tabular}{|c|c|c|c|c|c|c|}
\hline Implant Height & Type of Radiograph & Mean & Std. Deviation & $\mathrm{t}$ & df & Sig. \\
\hline \multirow{2}{*}{$8 \mathrm{~mm}$} & $\mathrm{PA}$ & -1.31000 & .41119 & -9.558 & 8 & .000 \\
\hline & OPG & -2.18000 & .60573 & -11.381 & 9 & .000 \\
\hline \multirow{2}{*}{$10 \mathrm{~mm}$} & PA & -1.27621 & 1.47346 & -6.596 & 57 & .000 \\
\hline & OPG & -2.63947 & .75673 & -26.334 & 56 & .000 \\
\hline \multirow{2}{*}{$11.5 \mathrm{~mm}$} & PA & -1.56368 & 1.45883 & -4.672 & 18 & .000 \\
\hline & OPG & -3.08429 & .73094 & -19.337 & 20 & .000 \\
\hline \multirow{2}{*}{$13 \mathrm{~mm}$} & PA & -1.65000 & 1.27047 & -5.510 & 17 & .000 \\
\hline & OPG & -1.59762 & .95986 & -7.627 & 20 & .000 \\
\hline
\end{tabular}

Table 3: Dimensional Magnifications between Various Implant Widths

\begin{tabular}{|c|c|c|c|c|c|c|}
\hline Implant Width & Type of Radiograph & Mean & Std. Deviation & $\mathrm{t}$ & df & Sig. \\
\hline \multirow{3}{*}{$3.5 \mathrm{~mm}$} & PA & -.1666 & .25558 & -4.833 & 54 & .000 \\
\hline & BW & -.30583 & .38261 & -3.916 & 23 & .001 \\
\hline & OPG & -.70870 & .48087 & -10.830 & 53 & .000 \\
\hline
\end{tabular}




\begin{tabular}{|c|c|c|c|c|c|c|}
\hline \multirow[t]{3}{*}{$4.3 \mathrm{~mm}$} & BW & -.12190 & .19018 & -4.881 & 57 & .000 \\
\hline & OPG & -.92182 & .58246 & -10.498 & 43 & .000 \\
\hline & PA & -.11727 & .08603 & -4.521 & 10 & .001 \\
\hline \multirow[t]{2}{*}{$5 \mathrm{~mm}$} & BW & -.15476 & .09097 & -7.796 & 20 & .000 \\
\hline & OPG & -.95545 & .51424 & -6.162 & 10 & .000 \\
\hline
\end{tabular}

To find out whether the maxilla or mandible is more affected with distortion, the maxilla and mandible were looked at separately. In all types of radiographs there was a significance difference between actual sizes and radiographic measurements when it comes to height and width in both maxilla and mandible $(\mathrm{P}<0.05)$. The radiographic magnification was more in maxilla than mandible for both $\mathrm{PAs}$ and OPGs. The anterior region in PAs had the greatest mean magnification $(2.16 \mathrm{~mm})$ in regard to the height, but in OPGs the posterior region had the greatest mean magnification $(3.03 \mathrm{~mm})$ in regard to the height. The mean width magnification was greatest in OPGs anteriorly and posteriorly in the maxilla $(0.78 \mathrm{~mm})$. On the other hand, the mean width magnification in the mandible was greater than the maxilla in BWs $(0.17 \mathrm{~mm})$. [Table 4]

Table 4: Dimensional Magnifications between Maxillary and Mandibular Implants

\begin{tabular}{|c|c|c|c|c|c|c|}
\hline & Type of Radiograph & Mean & Std. Deviation & $\mathrm{t}$ & df & Sig. \\
\hline \multirow{3}{*}{ Height Magnification on Maxilla } & PA & -1.66518 & 1.52279 & -8.183 & 55 & .000 \\
\hline & OPG & -2.50155 & .96406 & -23.782 & 83 & .000 \\
\hline & PA & -.15804 & .28953 & -4.085 & 55 & .000 \\
\hline Width Magnification on Maxilla & OPG & -.77548 & .53981 & -13.166 & 47 & .000 \\
\hline \multirow{3}{*}{ Height Magnification on Mandible } & PA & -1.07021 & 1.09460 & -6.774 & 47 & .000 \\
\hline & OPG & -2.27042 & .76083 & -20.675 & 47 & .000 \\
\hline & PA & -.14583 & .20629 & -4.898 & 47 & .000 \\
\hline Width Magnification on Mandible & OPG & -.73396 & .58544 & -8.686 & 47 & .000 \\
\hline
\end{tabular}

\section{Discussion}

This study was performed to find out the amount of image distortion associated with different digital radiographs and the reliability of the SIDEXIS software.

All the PAs and BWs measured in this study were taken using the bisecting angle technique, which might lead to a higher degree of distortion and magnification, which is supported by the findings of (Bilhan, Geckili et al. 2015) where they compared multiple dental radiographic techniques and found the parallel technique to be more precise than the bisecting angle technique.

The results of this study demonstrated that the measured distance on PAs was the closest to the actual dimensions of the implant fixture regardless to implantation site. (Wakoh, Harada et al. 2006)'s study found that measurement accuracy of standardized PAs was almost the same as, or better than, that of CT scan images, this is probably due to the sharpness and resolution of images obtained with standardized PA radiography.

OPGs showed the greatest measurement deviation from the actual dimensions of implant. It showed magnification in the radiographic length compared to the actual size by $2.74 \mathrm{~mm}$, that is in concordance with the finding of the previous studies done by (Schropp, Stavropoulos et al. 2009) where the difference mean was $1.25 \mathrm{~mm}$, but runs contrary to (Kayal 2016)'s study, where the radiographical length of the implant was examined and the measurements of the sample overall were decreased by $0.4 \mathrm{~mm}$ compared to the actual size. A study conducted by (Langlois Cde, Sampaio et al. 2011) revealed that PAs on average, overestimated the actual measurements from dry hemi-mandible, while OPGs underestimated the real values. The greatest disadvantage of OPG radiography is its insufficient resolution for anatomical details, which can be overcome by the use of complementary PAs (Dharmar 1997).

In all radiographic techniques there was difference in dimensions between the maxilla and mandible, among OPGs the maxilla had more distortion in height and width, this is in line with the findings of (Kim, Park et al. 2011). Regarding distortion in width, the curvature of the premaxilla which can't be represented by the X-ray machine might influence and effects on horizontal dimension. On the other hand, the difference in length, which was higher, is probably due to the angulations of the implants measured, which follows the apico-coronal accesses of the premaxilla and anterior mandible, in relation to the floor when the occlusal plane is parallel to it (Kayal 2016). (Kim, Park et al. 2011) measured implant sizes in the mandible and found that the magnification is higher in the anterior region in comparison to other areas, opposite to our finding where the posterior region had the maximum distortion, and the study done by (Park 2010) where it was found that the lower anterior region showed the lowest magnification and the lower premolar area generated the highest value.

The effect of implant length on magnification in OPGs was evaluated and it was found that the lowest value of magnification from the group with implants having longest length (Park 2010) which is in correspondence to our results where the longest implant was $13 \mathrm{~mm}$. Among PAs the lowest value of magnification was from the group with implants having $10 \mathrm{~mm}$ heights, whereas in BWs the highest magnification was from the group with the smallest widths at $3.5 \mathrm{~mm}$. In PAs, the maxilla had the greatest value of magnification regarding the height which was in the anterior region. whereas among BWs the magnification was the maximum in the mandible.

Several factors may explain the differences in measurements found in our study. One of them is the calibration method that can impact the accuracy and reproducibility obtained from digital radiographs. (Gotfredsen, Schropp et al. 2005) found that calibration with dedicated software had a greater impact on the anterior and premolar regions than on the molar region.

When comparing OPGs' findings in our software with PorDios for Windows software, we found the average distortion in height was 2.74mm and $0.97 \mathrm{In}$ width while in PorDios were $1.3 \mathrm{~mm}$ in and $1.2 \mathrm{~mm}$ respectively (Gotfredsen, Schropp et al. 2005).

Another factor is the positioning errors of the implants, several studies have considered positioning errors as a significant factor that could influence radiographic magnification (Yeo, Freer et al. 2002). (Schulze, Krummenauer et al. 2000) also reported that mandibular molars are usually better seen than other teeth on a panoramic radiograph and positioning errors have a less profound effect on these teeth.

(Schropp, Stavropoulos et al. 2009)'s study evaluated the effect of three implant systems Straumann AG, Nobel Biocare and BIOMET $3 \mathrm{i}$, it revealed that only small differences were found between the different implant systems and the Straumann system seemed less affected by the calibration methods.

Finally, the study done by (Park 2010) found that submerging the implant can be one of the factors of increased radiographic distortion compared to non-submerged implant. 


\section{Conclusion}

Digital OPG, PA and Bitewing radiographic techniques are reliable for performing vertical and horizontal linear measurements for dental implants, and PAs have the highest linear measurement precision with dose limitation available. Additionally, SIDEXIS software provided accurate results and can be considered as a reliable method for measuring root form implants in the studied region.

\section{References}

[1] Bilhan, H., O. Geckili, S. Arat Bilhan, F. Aycicek, B. Albayrak, P. Bozbulut and F. Unalan (2015). "The comparison of the precision of different dental radiographic methods in mandibular peri-implantary measurements: an in vitro study." J Istanb Univ Fac Dent 49(1): 1-9. https://doi.org/10.17096/jiufd.55134.

[2] Carmichael, F. A., P. N. Hirschmann, B. Scaife, L. Sheard and A. Mackenzie (2000). "A comparison of the diagnostic utility of two image receptors for panoramic radiography." Dentomaxillofac Radiol 29(1): 57-60. https://doi.org/10.1038/sj.dmfr.4600495.

[3] Dharmar, S. (1997). "Locating the mandibular canal in panoramic radiographs." Int J Oral Maxillofac Implants 12(1): 113-117.

[4] Gotfredsen, E., L. Schropp and A. Wenzel (2005). Software used to predict implant size from digital panoramic radiographs. https://doi.org/10.1016/j.ics.2005.03.363.

[5] Kayal, R. A. (2016). "Distortion of digital panoramic radiographs used for implant site assessment." J Orthod Sci 5(4): 117-120. https://doi.org/10.4103/2278-0203.192113.

[6] Kim, Y. K., J. Y. Park, S. G. Kim, J. S. Kim and J. D. Kim (2011). "Magnification rate of digital panoramic radiographs and its effectiveness for pre-operative assessment of dental implants." Dentomaxillofac Radiol 40(2): 76-83. https://doi.org/10.1259/dmfr/20544408.

[7] Langlois Cde, O., M. C. Sampaio, A. E. Silva, N. P. Costa and M. I. Rockenbach (2011). "Accuracy of linear measurements before and after digitizing periapical and panoramic radiography images." Braz Dent J 22(5): 404-409. https://doi.org/10.1590/S0103-64402011000500010.

[8] Machtei, E. E., H. Zigdon, L. Levin and M. Peled (2010). "Novel ultrasonic device to measure the distance from the bottom of the osteotome to various anatomic landmarks." J Periodontol 81(7): 1051-1055. https://doi.org/10.1902/jop.2010.090621.

[9] McDavid, W. D., S. B. Dove, U. Welander and G. Tronje (1993). "Dimensional reproduction in direct digital rotational panoramic radiography." Oral Surg Oral Med Oral Pathol 75(4): 523-527. https://doi.org/10.1016/0030-4220(93)90182-4.

[10] Park, J. B. (2010). "The evaluation of digital panoramic radiographs taken for implant dentistry in the daily practice." Med Oral Patol Oral Cir Bucal 15(4): e663-666. https://doi.org/10.4317/medoral.15.e663.

[11] Sahiwal, I. G., R. D. Woody, B. W. Benson and G. E. Guillen (2002). "Radiographic identification of threaded endosseous dental implants." J Prosthet Dent 87(5): 563-577. https://doi.org/10.1067/mpr.2002.124430.

[12] Samawi, S. S. and P. H. Burke (1984). "Angular distortion in the orthopantomogram." Br J Orthod 11(2): 100-107. https://doi.org/10.1179/bjo.11.2.100.

[13] Sanderink, G. C., W. N. Visser and E. W. Kramers (1991). "The origin of a case of severe image distortion in rotational panoramic radiography." Dentomaxillofac Radiol 20(3): 169-171. https://doi.org/10.1259/dmfr.20.3.1808003

[14] Schropp, L., A. Stavropoulos, E. Gotfredsen and A. Wenzel (2009). "Calibration of radiographs by a reference metal ball affects preoperative selection of implant size." Clin Oral Investig 13(4): 375-381. https://doi.org/10.1007/s00784-009-0257-5.

[15] Schulze, R., F. Krummenauer, F. Schalldach and B. d'Hoedt (2000). "Precision and accuracy of measurements in digital panoramic radiography." Dentomaxillofac Radiol 29(1): 52-56. https://doi.org/10.1038/sj.dmfr.4600500.

[16] Tyndall, D. A. and S. L. Brooks (2000). "Selection criteria for dental implant site imaging: a position paper of the American Academy of Oral and Maxillofacial radiology." Oral Surg Oral Med Oral Pathol Oral Radiol Endod 89(5): 630-637. https://doi.org/10.1067/moe.2000.106336.

[17] Wakoh, M., T. Harada, T. Otonari, M. Otonari-Yamamoto, M. Ohkubo, Y. Kousuge, N. Kobayashi, S. Mizuta, H. Kitagawa and T. Sano (2006). "Reliability of linear distance measurement for dental implant length with standardized periapical radiographs." Bull Tokyo Dent Coll 47(3): 105115. https://doi.org/10.2209/tdcpublication.47.105.

[18] Yeo, D. K., T. J. Freer and P. J. Brockhurst (2002). "Distortions in panoramic radiographs." Aust Orthod J 18(2): $92-98$. 